\title{
Low Reynolds number flow and heat transfer experiments in 7-rod vertical bundles
}

\author{
A.K. Mohanty ${ }^{a}$, S.C. Haldar ${ }^{a}$ and S. Sengupta ${ }^{b}$ \\ ${ }^{a}$ Department of Mechanical Engineering, Indian Institute of Technology, Kharagpur-721302, India \\ ${ }^{b}$ School of Engineering University of Michigan, Dearborn, MI, USA
}

Received 1 February 1993

\begin{abstract}
Experiments have been conducted in 7-rod vertical bundles within circular shell. Buoyancy supports the forced flow in one and opposes it in the other. The Reynolds number range nominally covered 900 to 30000 ; but no distinct transition was observed. The behaviour is explained through a porous medium model. Procedures for estimating bundle-average permeability and fRe values on the basis of porosity have been illustrated for the case of forced flow. Although friction was increased in downflow and decreased in upflow due to buoyancy, change in thermal transport rate was not discerned within $\mathrm{Gr} / \mathrm{Re}= \pm 40$.
\end{abstract}

\section{Introduction}

Thermal hydraulic studies in the rod-bundle geometry have received considerable attention in recent years from the considerations of the safety of nuclear reactors. Whereas the flow regime in the normal operating conditions of a reactor is high Reynolds number turbulent, the accident condition is characterized by low flow and degenerated transport rates.

Laminar analyses for the rod-bundles have been carried out for sub-channels models [1-3] applicable in zones far removed from the boundary, or on the basis of symmetry sectors $[4,5]$ accounting for the influence of the bounding shell. The latter geometry is relevant to small diameter heat exchangers and the CANDU type of reactors. Whereas the sub-channel geometry is definable through a simple pitch to diameter ratio $(P / D)$, the narrower geometry requires an additional parameter of wall to diameter ratio $(W / D)$. It has been observed $[2,4]$ that the transport rate values are strongly $P / D$ ratio sensitive, especially when the geometry is narrow and when buoyancy effects are present.

Analyses of turbulent transport rates [6-8] have been made using both algebraic and higher order closure models. These stress models being fundamentally for high Reynolds number flow, application of so-derived transport rate values to accident condition is a suspect. This apprehension is borne out by experimental results $[9,10]$, sparse as those are.

Fluid flow and heat transfer experiments have been performed for sub-channel geometries with rods in triangular and square pitches $[9,10]$. Bundles with rod numbers varying from 7 to 61 in circular and hexagonal [9] shells have also been reported. The results ordinarily pertain to high Reynolds number; and the laminar theory is practically not verified by experiments. Rehme's [9] measurements with a variety of bundle size indicate that a laminar-turbulent transition is not distinct. A numerical study of Yang [12] indicated that the influence of buoyancy is not pronounced at $\mathrm{Gr} / \mathrm{Re}$ $<100$. A survey of the literature together with the practical requirements points to the following.

The accident condition, which is the impetus of recent thermal-hydraulic studies, may not really be in the laminar regime. Conclusions dependent on $P / D$ ratio, buoyancy or the like arrived at analytically, from laminar considerations, could be misleading. The rodbundle geometry being complex, the laminar regime may cover vanishingly small range of Reynolds number. Both the laminar conclusions and high $\mathrm{Re}$ turbulent information are unlikely to apply to safety studies.

We have undertaken the present study with a view to generate information over a range of Reynolds number which are low compared to the normal operat- 
ing conditions; hence the title. Experiments have been conducted in 7-rod vertical bundles in circular shells with up and down flows. Transport rates under low Reynolds number condition in rod-bundles of arbitrary configuration has been modelled as porous medium phenomena. Literature information on sub-channels have been used for correlating with bundle porosity.

\section{Experiments}

Fluid flow and heat transfer studies were carried out experimentally. Forced flow of air was caused through 7-rod test sections representing finite arrays.

\subsection{Experimental set-up}

Three identical sets of 7-rod bundle were made for fluid flow and heat transfer measurements. The rods were each $12 \mathrm{~mm}$ OD stainless steel tubes, $1200 \mathrm{~mm}$ long held between end plates. Each tube was filled with ceramic modules having six axial holes, four of which were used for passing nichrome wire heating elements. The other two holes were used for thermocouple wires soldered from the tube inner wall, thus avoiding disturbance to the flow field. The wires were taken out through asbestos caps sealing the tube ends, and were made to pass through the end plates without obstructing the flow passage. Seven rods were assembled inside a $54.5 \mathrm{~mm} \mathrm{ID,} 4 \mathrm{~mm}$ thick plexiglass circular shell.

The sections of the heater rod, end plates and the rod-arrangements are shown in Fig. 1. It will be noted from the figure that three sections were arranged in series: two verticals and one horizontal, separated by plain pipe sections used for locating traversing probes. Under heating conditions, the acrylic shell walls were covered with asbestos rope to avoid heat losses. The assembled three test sections in series were connected to a two-stage blower for air flow in vertically down, horizontal and up directions. A by-pass and gate-valve arrangement controlled the air flow rate which was monitored by an orifice plate. The test sections were isolated against vibration from the blower by coupling through a leather-bellow.
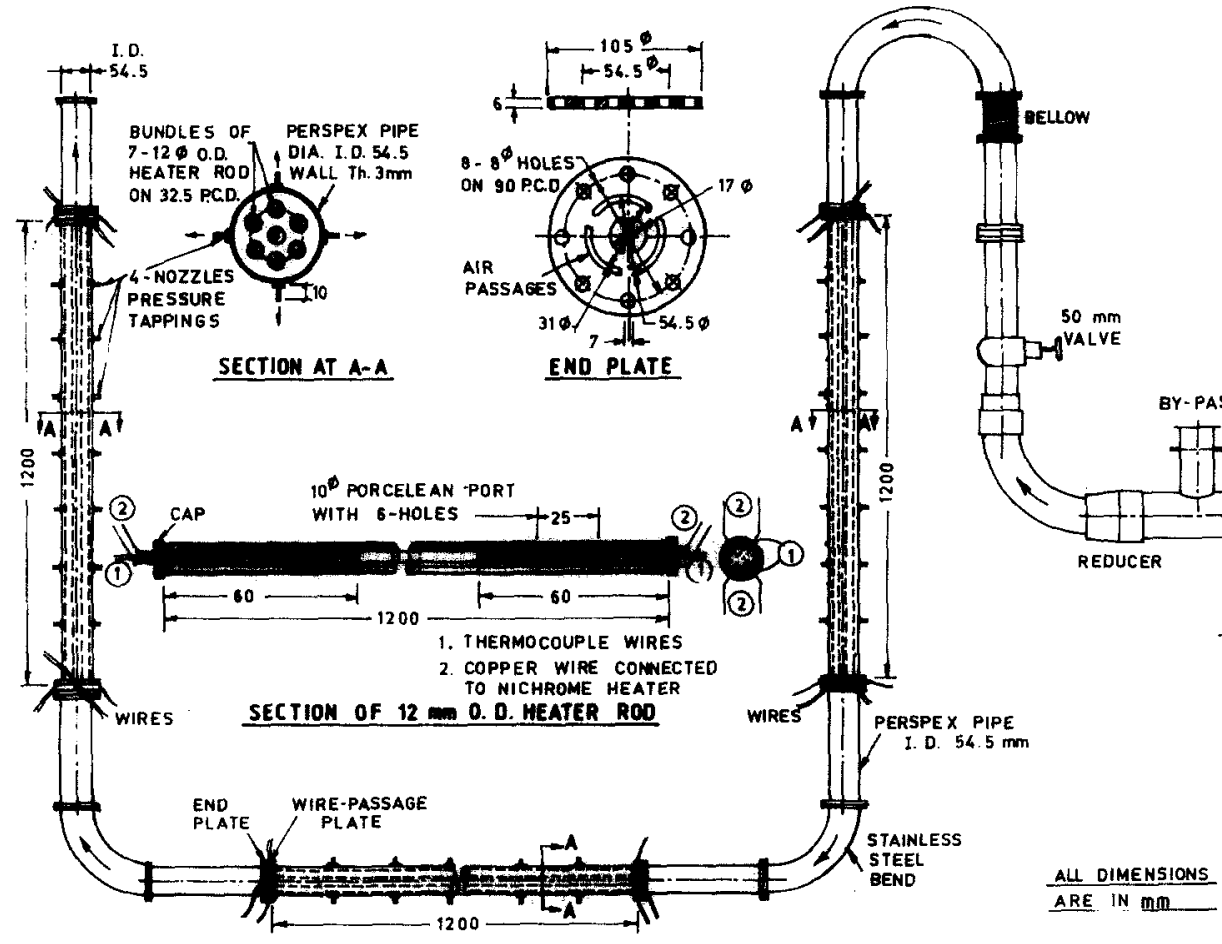

Fig. 1. Experimental set-up for combined forced and free convection studies in rod bundles. 


\subsection{Instrumentation}

Apart from the orifice plate, the flow rate was estimated from a pitot traverse in the plain sections. Micrometric traversing gear and a micromanometer were used for the purpose. Copper-constantan thermocouples were traversed for the estimations of inlet and outlet bulk temperature of air for each test-section. The pitot tube was not very effective when the flow rate was low. The orifice plate also is a suspect at low flow rates. The flow rate was, therefore, checked through enthalpy change:

$\dot{m} C_{\mathrm{p}}\left(T_{\mathrm{b} \text { out }}-T_{\mathrm{b} \text { in }}\right)=Q_{\text {in }}-Q_{\text {leakage }}$.

In order to estimate the heat loss through the $4 \mathrm{~mm}$ thick outer plexiglass shell, measurements were made with and without asbestos rope insulation. Practically no difference was noted, and the heat leakage was discounted. Four static pressure tappings were made at $90^{\circ}$ angular spacings at $150 \mathrm{~mm}$ axial intervals along the outer-shells. The four tappings at a section were joined in parallel for the measurement of cross-sectional average static pressure.

Experiments were carried out for one section at a time. Each of the heater rods was supplied electric power independently through an auto-transformer and precision volt and ampere meters from a stabilized power source. The built-in thermocouples provided temperature information for each of the rods, $20 \mathrm{~mm}$ from the inlet, mid-length and $20 \mathrm{~mm}$ before the exit of a test section. Power supply to each of the rods was maintained equal, and a condition of uniform heat flux was simulated.

\subsection{Uncertainty analysis}

Each of the experimentally determined parameter, e.g. flow rate, pressure, temperature, power input was subjected to uncertainty analyses. The uncertainties in pipe and rod diameters and lengths, as well as the accuracy limits of the instruments used were accounted for. Analyses carried out follows the method of Kline and McClintock, as quoted by Holman [11], yielded typical uncertainty values of $0.5 \%$ for pressure, $1.8 \%$ for temperature, $4.2 \%$ for heat flux and $3.1 \%$ for flow rate. Accounting further 0.8 and 0.5 percents for pipe and rod diameters, the uncertainty for friction factor was $3.1 \%$, Reynolds number $3 \%$ and a value of $5 \%$ for Nusselt number for $\operatorname{Re}$ nominally more than $1 \times 10^{4}$. At lower flow rates, the uncertainties were, on the average, $2 \%$ higher.

\section{Results}

Pressure drop measurements across consecutive 150 $\mathrm{mm}$ tappings were carried out on the two vertical legs. The first set of measurements were without heating. Pressure drops across each equal length were practically the same, signifying that the flow was hydrodynamically developed in each section. This was so, because the test sections were preceded by several pipe sections, and the net flow area through a bundle was quite small compared to the wetted surface. Even then, we discounted the pressure readings of the first four tappings being influenced by possible entry effects. Likewise, the temperature reading at the inlet of a rod was kept out of the calculations for fully developed heat transfer results.

\subsection{Isothermal friction factor}

The experimental Fanning's friction factor is estimated from the measured static pressure drop as

$f=\frac{D_{h}}{2 \rho u_{\mathrm{av}}^{2}}\left(-\frac{\Delta p}{\Delta L}\right)$.

Acceleration effects are absent at fully developed condition, and the hydrostatic effect, if any, was eliminated by the differential measurement of pressure. For unheated tests, the value of density $\rho$ was calculated on the basis of the measured pressure and temperature at inlet to the test section. The inlet condition was also used for the estimation of Reynolds number. The bundle hydraulic diameter is evaluated as $D_{\mathrm{h}}=4\left(D_{\mathrm{s}}^{2}-\right.$ $\left.7 \mathrm{D}^{2}\right) /\left(D_{\mathrm{s}}+7 D\right)$.

The variation of friction factor with Reynolds number is shown in Fig. 2. The measurements covered a nominal Re values between 900 to 30000 . However, a clear transition from laminar to turbulent regime was not discernible. Similar observations were made by Rehme [9]. It is observed from Fig. 2, that deviations between the curve correlating the measured $f$ and its laminar estimates agree within less than $10 \%$ for $\mathrm{Re}$ less than 700. This value of $\mathrm{Re}$ may, therefore, be taken as the laminar limit.

\subsection{Buoyancy effects on friction factor}

Pressure drop measurements were carried out in both the vertical legs with heat input. The temperature variations over the measuring length was estimated by linear interpolation of the values between the inlet and outlet of a test section with uniform heat input. The 


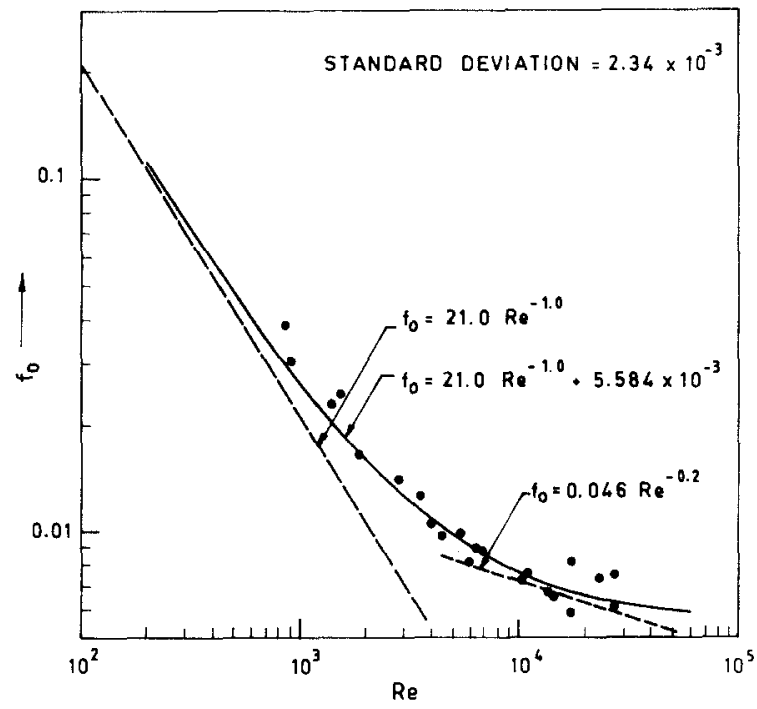

Fig. 2. Isothermal friction factor, 7-rod bundles.

average values of $\rho$ and $u_{\mathrm{av}}$ over the measuring section were calculated accounting for temperature variations, and used for $f$ and $\mathrm{Re}$ estimations.

The flow is downwards in the leg nearest to the blower, and buoyancy opposes the forced flow. An increased pressure gradient is reflected through a higher friction factor, Fig. 3. A supporting effect is observed in the left hand side of Fig. 3 for the upward flow in the far vertical leg. These observations were made at Reynolds number in the range of 900-2000. The maximum changes in friction factor are within $10 \%$ of the isothermal value for $(\mathrm{Gr} / \mathrm{Re})$ ratio in the

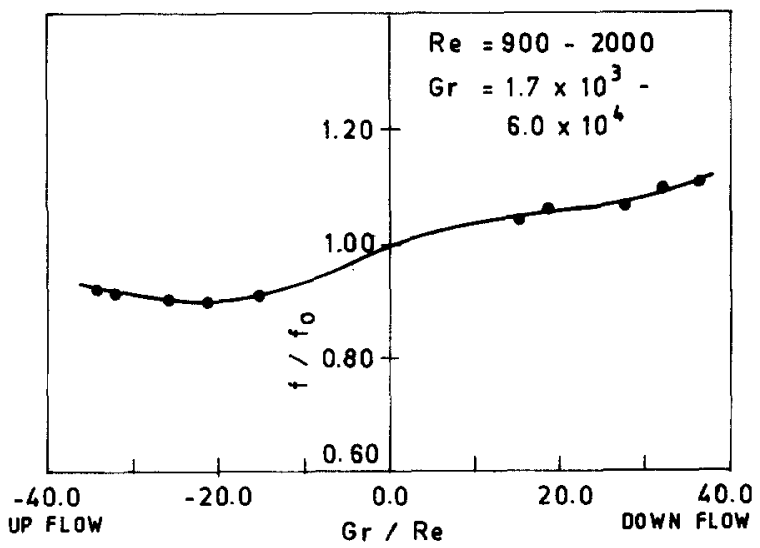

Fig. 3. Buoyancy effects on friction factor. neighbourhood of 40 in both supporting and opposing flows. Note that a $(\mathrm{Gr} / \mathrm{Re})$, not $\left(\mathrm{Gr} / \mathrm{Re}^{2}\right)$, variable is used because the flow was deemed developed [4].

Unlike in the case of a peripherally heated tube, the buoyancy effects in the rod-bundle geometry are predominant away from the shell-wall. It is convenient to visualize buoyancy as a superimposed pressure gradient on the bulk flow, than in terms of the shell-wall velocity gradient [16]. The buoyancy thus causes an adverse pressure gradient in the downflow and supports an upflow in the rod-bundle geometry. This explains the observations in Fig. 3 which are also supported by the laminar analysis of Yang [12]. Studies in ref. [4] further indicated that the buoyancy influenced friction factor behaviour can also change with the bundle pitch to diameter ratio.

\subsection{Heat transfer}

The axial variations of heat transfer along the rods were monitored from rod surface and fluid bulk temperature measurements at the inlet, mid section and exit from a test bundle. The mid and end section temperature differences $\left(T_{\mathrm{w}}-T_{\mathrm{b}}\right)$ were equal within a maximum variation of $8 \%$. The inlet end temperature differences were lower by factors of 2 to 3 compared to the exit, apparently due to the thermal entry effects. Far downstream from the inlet, or in a continuous loop, the transport rates are low and longitudinally constant. With uniform heat input, this results in a constant value of the rod to fluid temperature difference [17]. Therefore, the $\left(T_{\mathrm{w}}-T_{\mathrm{b}}\right)$ value at the exit end was used for estimating the fully developed heat transfer rate:

$h=q^{\prime \prime} /\left(T_{\mathrm{w}}-T_{\mathrm{b}}\right)$

The $\left(T_{\mathrm{w}}-T_{\mathrm{b}}\right)$ value for the central rod was, in general, 40 to $50 \%$ higher than that for the peripheral rods. Typically at a $\operatorname{Re}=11600$, and low heat input of $q^{\prime \prime}=$ $421.7 \mathrm{~W} / \mathrm{m}^{2}$ where the buoyancy effect at $\mathrm{Gr} / \mathrm{Re}=$ 3.23 can be ignored, the central rod temperature was $323.6 \mathrm{~K}$, that of a peripheral rod $320.2 \mathrm{~K}$, and the fluid bulk temperature $311.1 \mathrm{~K}$ at the exit end.

Nusselt number was defined on the hydraulic diameter and property values calculated at the average of the heated surface and fluid temperatures. Variations of $\mathrm{Nu}$ with Reynolds number for peripheral rods and the central rod are shown in Fig. 4. The bundle average values are plotted in Fig. 5. 


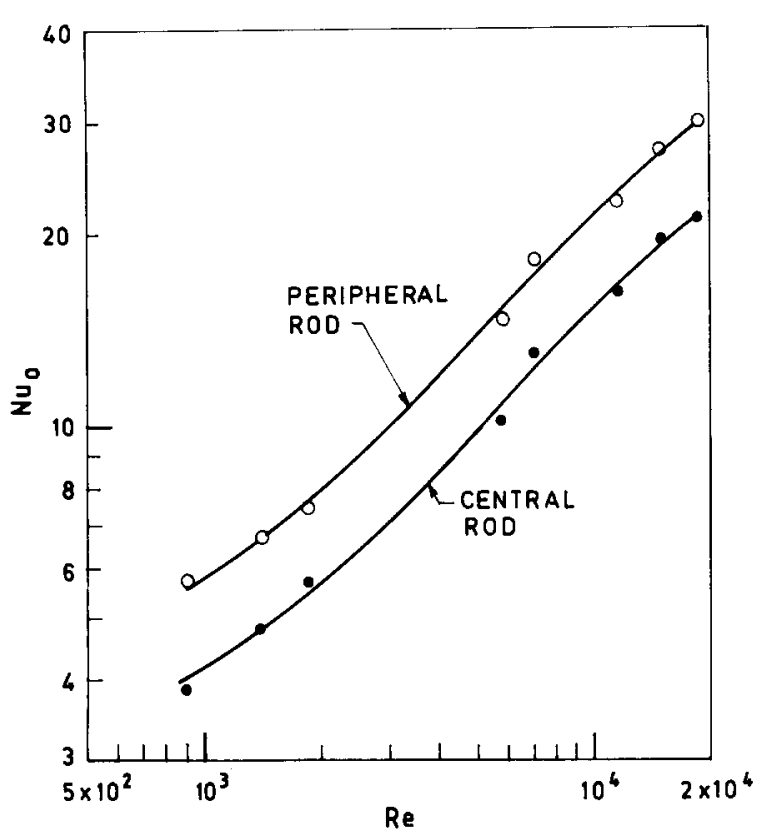

Fig. 4. Heat transfer rate variation for central and peripheral rods, forced flow.

With increasing heat flux, the $\mathrm{Gr} / \mathrm{Re}$ value was raised upto \pm 37 . It would be seen from Fig. 6 , that buoyancy effects on Nusselt number are marginal in the range of experimental parameters. The thermal transport tends to decrease, if any, in both up and

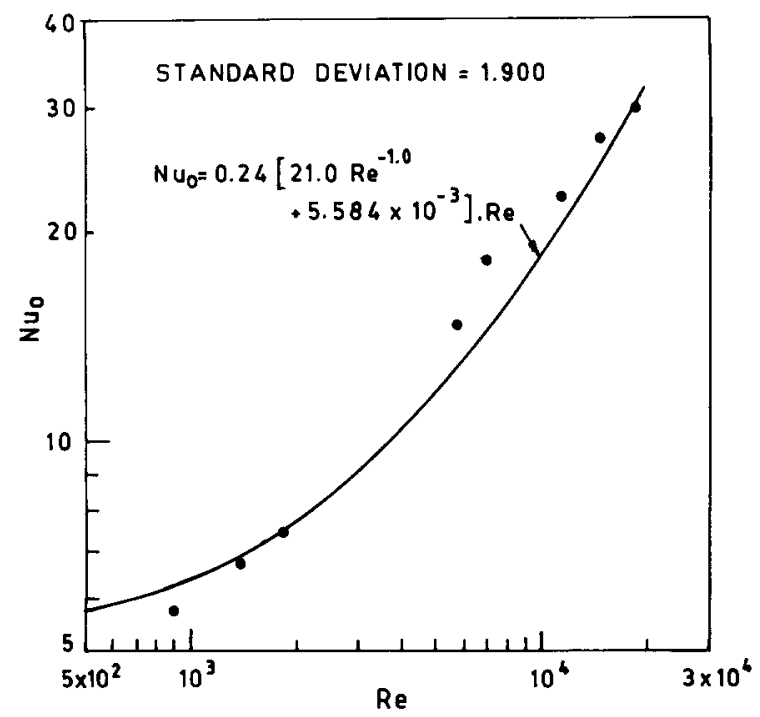

Fig. 5. Bundle average heat transfer rates, forced flow.

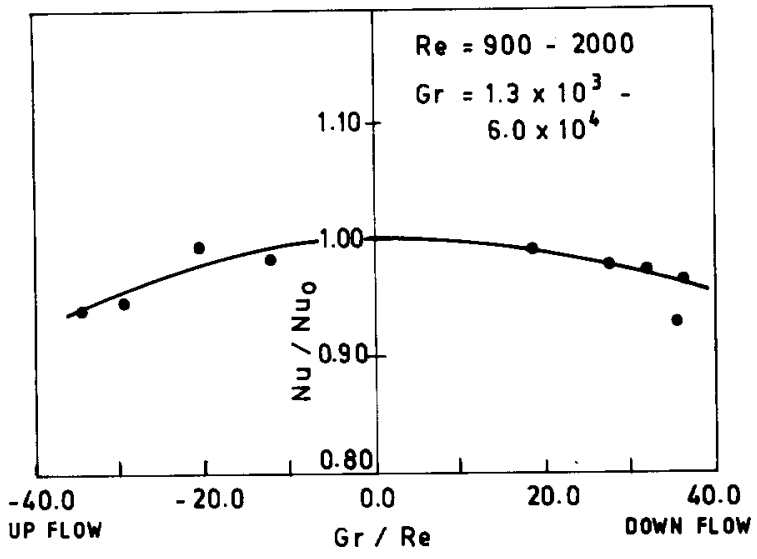

Fig. 6. Buoyancy effects on heat transfer.

down flow. This is in agreement with the experimental observation of Kim and El-Genk [10] for $\mathrm{Gr} / \mathrm{Re}^{2}<1$.

\section{Analysis of experimental results}

The experimentally determined friction factor results in Fig. 2 are compared with the two limiting situations: laminar and turbulent. Since, at a given cross-section in a rod-bundle, several flow passages exist with differing cross-sectional areas, the flow through one may remain laminar whereas it may be turbulent through another, especially at moderate to low cross-sectional flow rate conditions. We believe that these variations reflect through a gross behaviour that is continuous from low to moderate Reynolds number, as seen in Fig. 2.

\subsection{Porous model}

In order to quantify the behaviour, we propose that the bundle cross-section be treated as a porous medium. A bundle is a combination of square and/or triangular sub-channels and curved or straight boundary channels [1]. Laminar friction factor values are available in the literature for these constituent passages $[13,14]$. We have attempted to summarize these information through porosity $\epsilon$ which merge the $P / D$ and $W / D$ identities.

\subsubsection{Porosity}

Porosity $\epsilon$ is defined as the ratio of the volume of the void passage, $V_{\mathrm{v}}$ to the total volume, $V$ [15].

$\left.\begin{array}{l}V_{\mathrm{v}}=V-V_{\mathrm{s}} \\ \epsilon=1-V_{\mathrm{s}} / V\end{array}\right\}$

where $V_{\mathrm{s}}=$ volume of the solid. 


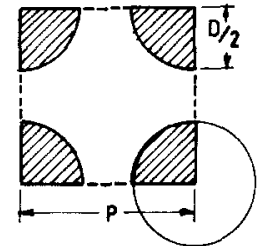

SQUARE PITCH

INTERIOR SUB-CHANNELS

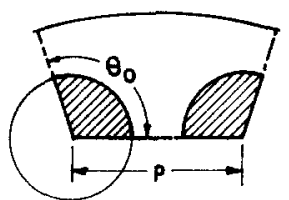

CURVED BOUNDARY

WALL SUB-CHANNELS

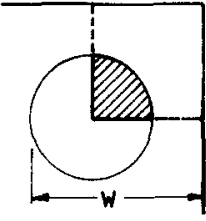

SQUARE CLUSTER
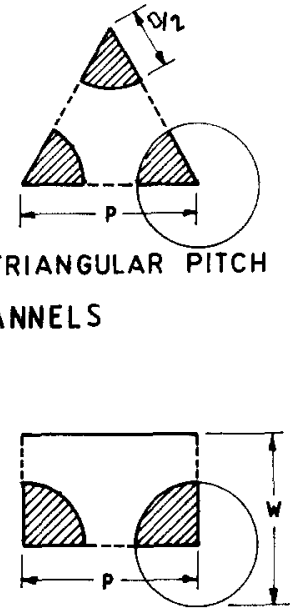

TRIANGULAR PITCH

STRAIGHT BOUNDARY

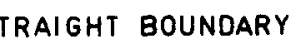

\section{CORNER SUB-CHANNELS}

Fig. 7. Sub-channel geometries.

The estimation of porosity is carried out for different sub-channels by a reference to Fig. 7. Additionally, a measure of the wetted surface offering shear resistance is the specific surface area $S$ calculated per unit volume. Expressions for these parameters are given for different type of subchannels.

\section{Interior sub-channels}

Square:

$$
\left.\begin{array}{l}
V=P^{2}, \quad V_{\mathrm{s}}=\frac{\pi}{4} D^{2}, \quad \epsilon=1-\frac{\pi}{4}(\mathrm{PDR})^{-2}, \\
\mathrm{SD}=\pi(\mathrm{PDR})^{-2}
\end{array}\right\}
$$

Triangular:

$$
\begin{aligned}
& V=\frac{\sqrt{ } 3}{4} P^{2}, V_{\mathrm{s}}=\frac{\pi}{8} D^{2}, \epsilon=1-\frac{\pi}{2 \sqrt{3}}(\mathrm{PDR})^{-2}, \\
& \mathrm{SD}=\frac{2 \pi}{\sqrt{3}}(\mathrm{PDR})^{-2} .
\end{aligned}
$$

Wall sub-channels

Straight:

$$
\left.\begin{array}{l}
V=P\left(W-\frac{D}{2}\right), \quad V_{\mathrm{s}}=\frac{\pi}{8} D^{2}, \\
\epsilon=1-\frac{\pi}{8}\left[\operatorname{PDR}\left(\mathrm{WDR}-\frac{1}{2}\right)\right]^{-1}, \\
\mathrm{SD}=\left(\frac{\pi}{2}+\operatorname{PDR}\right)\left[\operatorname{PDR}\left(\mathrm{WDR}-\frac{1}{2}\right)\right]^{-1} ;
\end{array}\right\}
$$

Curved:

When there are $n$ rods in the outer most ring,

$\theta_{0}=\pi\left(\frac{1}{n}+\frac{1}{2}\right)$

$V=\frac{1}{4}\left(\frac{\pi}{n} D_{\mathrm{s}}^{2}+P^{2} \tan \theta_{0}\right), \quad V_{\mathrm{s}}=\frac{D^{2} \theta_{0}}{4}$,

$\epsilon=1-\theta_{0}\left[\frac{\pi}{n} \mathrm{SDR}^{2}+\mathrm{PDR}^{2} \tan \theta_{0}\right]^{-1}$,

$\mathrm{SD}=4\left(\theta_{0}+\frac{\pi}{n} \mathrm{SDR}\right)\left[\frac{\pi}{n} \mathrm{SDR}^{2}+\mathrm{PDR}^{2} \tan \theta_{0}\right]^{-1}$

Calculations for the corner channels are made according to the geometry, see Fig. 7.

\subsubsection{Permeability}

The flow through a porous medium is described in terms of a superficial velocity $\bar{u}$ which is related to the pressure gradient through permeability $k$, and is defined by Darcy's law.

$\bar{u}=-\frac{k}{\mu} \frac{\mathrm{d} p}{\mathrm{~d} x}$.

An experimentally successful practice is the Kozeny-Carman capillary tube model for permeability which assumes $k S^{2}=c \epsilon^{3}$, where $S$ is the specific surface area per unit volume. For instance, $S=\pi D / P^{2}$ for a square sub-channel of pitch $P$. Through several trials and errors, we found that both the interior and wall sub-channels, accounting for $P / D$ and $W / D$, could be modelled through

$k S^{2}=a \epsilon^{m}$,

where $a$ and $m$ are to be determined for different class of sub-channels presented in Fig. 7.

The expression for $f \operatorname{Re}$ is

$f \operatorname{Re}=\frac{-\frac{\mathrm{d} p}{\mathrm{~d} x} D_{\mathrm{h}}}{2 \rho u_{\mathrm{av}}^{2}} \frac{\rho u_{\mathrm{av}} D_{\mathrm{h}}}{\mu}$ 
Table 1

Correlations for permeability of sub-channels $k S^{2}=a \epsilon^{m}$

\begin{tabular}{|c|c|c|c|c|}
\hline Arrangement & $a$ & $m$ & $\begin{array}{l}\text { Range } \\
\epsilon\end{array}$ & $\begin{array}{l}\text { Max. } \\
\text { deviation }\end{array}$ \\
\hline \multicolumn{5}{|l|}{ Interior sub-channels } \\
\hline Triangular & 0.095 & 2.012 & $0<\epsilon \leq 0.3$ & $2 \%$ \\
\hline Triangular & 0.184 & 2.424 & $0.3<\epsilon \leq 0.8$ & $3 \%$ \\
\hline Square & 0.084 & 1.273 & $0<\epsilon \leq 0.3$ & $0.5 \%$ \\
\hline Square & 0.159 & 1.826 & $0.3<\epsilon \leq 0.8$ & $2 \%$ \\
\hline \multicolumn{5}{|l|}{ Corner sub-channels } \\
\hline Hexagonal & 0.335 & 2.931 & $0<\epsilon \leq 0.8$ & $1 \%$ \\
\hline Square & 0.328 & 2.604 & $0<\epsilon \leq 0.8$ & $1.5 \%$ \\
\hline \multicolumn{5}{|l|}{ Wall sub-channels } \\
\hline Straight boundary & 0.268 & 2.458 & $0<\epsilon \leq 0.9$ & $1 \%$ \\
\hline \multicolumn{5}{|l|}{ Curved boundary } \\
\hline$\theta_{0}=120^{\circ}$ & 0.288 & 2.200 & $0<\epsilon \leq 0.8$ & $4 \%$ \\
\hline$\theta_{0}=105^{\circ}$ & 0.252 & 2.161 & $0<\epsilon \leq 0.8$ & $1 \%$ \\
\hline$\theta_{0}=100^{\circ}$ & 0.236 & 2.403 & $0<\epsilon \leq 0.8$ & $0.5 \%$ \\
\hline
\end{tabular}

where $u_{\text {av }}$ is the average velocity through the free or void space.

Application of Darcy's law, eq. (9), to eq. (11) yields

$f \operatorname{Re}=\frac{\bar{u}}{u_{\mathrm{av}}} \frac{D_{\mathrm{h}}^{2}}{2 k}$,

where the velocity ratio $\bar{u} / u_{\mathrm{av}}$ equals the porosity $\epsilon$ for mass conservation. Rewriting the hydraulic diameter $D_{\mathrm{h}}=4 A_{\mathrm{f}} / P_{\mathrm{w}}$ for a subchannel as

$D_{\mathrm{h}}=\frac{4 A_{\mathrm{f}} / A}{P_{\mathrm{w}} / A}=\frac{4 \epsilon}{S}$,

we get

$f \operatorname{Re}=\frac{\epsilon}{2 k}\left(\frac{4 \epsilon}{S}\right)^{2}=\frac{8 \epsilon^{3}}{k S^{2}}=C_{1}$

as the relationship between friction, porosity and permeability.

We estimated $\epsilon$ and $S$ for different kinds of subchannels and used the literature value for corresponding laminar $f \operatorname{Re}$ and arrived at the permeability, $k$, values which was, in turn, compared with eq. (10). A summary of this exercise is given in Table 1 , and representative results are shown in Figs. $8 \mathrm{a}, \mathrm{b}$ and $\mathrm{c}$ respectively for an interior, corner and wall sub-channel. The values of maximum deviations, cited alongside, attest the applicability of the correlations. The tabled results cover a wide variation of $P / D$ from 1.01 to 2 and $W / D$ from 1.05 to 2.0 .

\subsubsection{Superposition}

Given a sub-channel, we can estemate $\epsilon$ and $S$. We then use the information in Table 1 to generate the permeability value $k$. The permeability value for a bundle, consisting of several sub-channels, are then generated by superposition. The necessary conditions are: (i) the bundle average flow rate is summation of the sub-channel flow rates, and that (ii) an equal pressure gradient acts on each sub-channel as well as the bundle.

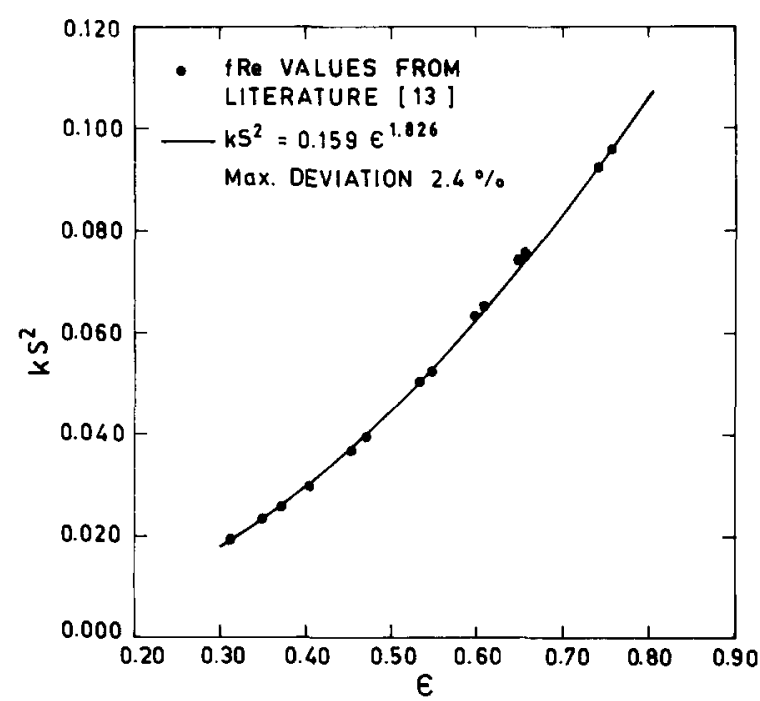

Fig. 8a. Permeability vs. porosity, square sub-channels. 


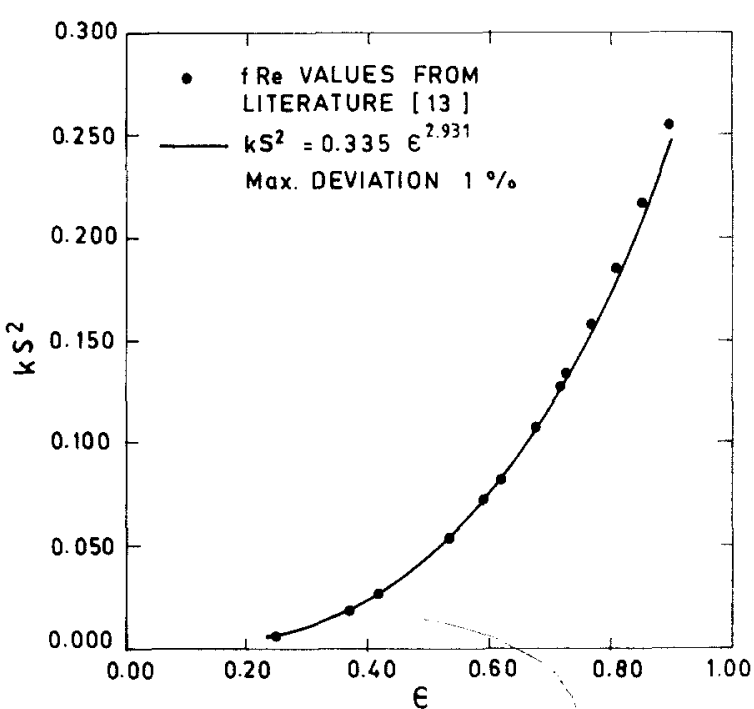

Fig. 8b. Permeability vs. porosity, corner sub-channel, hexagonal cluster.

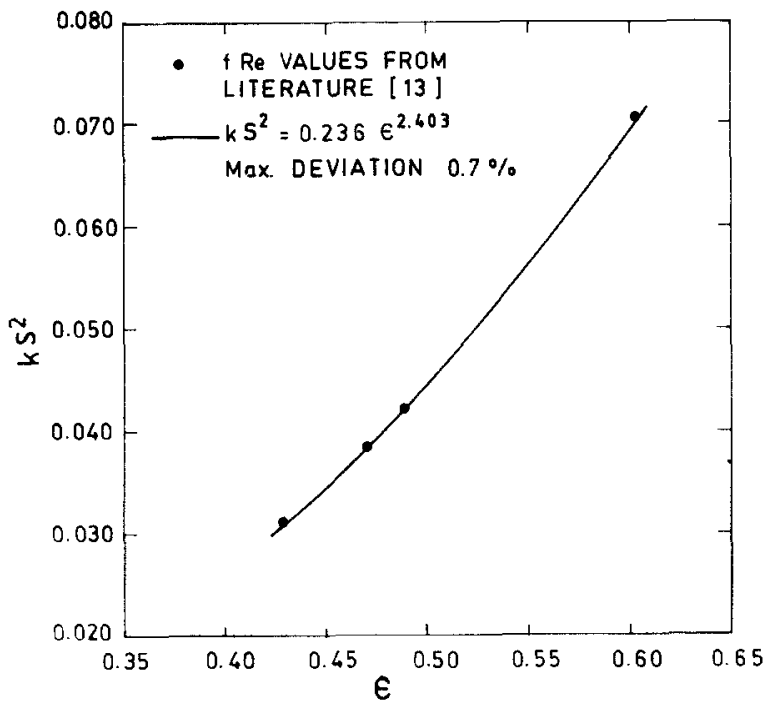

Fig. 8c. Permeability vs. porosity, wall sub-channel curved boundary $\theta_{0}=120^{\circ}$.

Hence

$A_{\mathrm{b}} \bar{u}_{\mathrm{b}}=\sum A_{\mathrm{i}} \bar{u}_{\mathrm{i}}$,

and

$\left(\frac{\mathrm{d} p}{\mathrm{~d} x}\right)_{\mathrm{b}}=\left(\frac{\mathrm{d} p}{\mathrm{~d} x}\right)_{i}$
Using Darcy's equation (9) in (14), we get

$A_{\mathrm{b}} \frac{k_{\mathrm{b}}}{\mu}\left(\frac{\mathrm{d} p}{\mathrm{~d} x}\right)_{\mathrm{b}}=\sum A_{i} \frac{k_{i}}{\mu}\left(\frac{\mathrm{d} p}{\mathrm{~d} x}\right)_{i}$, or

$k_{\mathrm{b}}=\frac{1}{A_{\mathrm{b}}} \sum A_{i} k_{i}$.

In otherwords, the bundle permeability is the areaaveraged permeabilities of the constitutent sub-channels.

The bundle average $f$ Re value is then readily obtained using eqs. (13). We have compared the porosity model results for a 7-rod bundle having wide variations in $P / D$ and $W / D$ values, with predictions reported in [1], see Kakac et al. [13], in Fig. 9. The maximum deviation is about $4 \%$. The agreement will be yet better when the number of rods in a bundle are increased.

\subsubsection{Laminar $f \mathrm{Re}$ for the experimental geometry}

We now propose to derive the laminar friction factor value for the experimental geometry, which offers six triangular and six wall sub-channels, through the porosity model. The geometrical values are: PDR $=1.3125, \mathrm{SDR}=4.5416, \theta_{0}=2 \pi / 3$.

Using expression (6) for the triangular sub-channel, we get $\epsilon=0.4735$ and $\mathrm{SD}=2.1058$. The sub-channel area is $A_{1}=0.7459 D^{2}$. The recommended values of $a=0.184$ and $m=2.424$ are read from Table 1 , and we evaluate $k_{1}=6.7757 \times 10^{-3} D^{2}$.

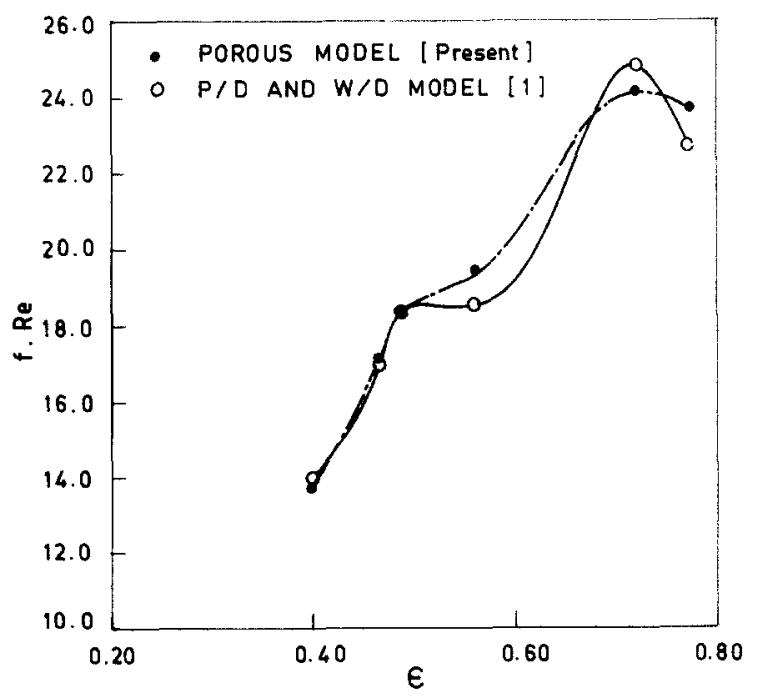

Fig. 9. $f$ Re values for circular clusters, 7-rod. 
The values for the wall subchannel are derived using expression (8): $\epsilon=0.7320, \mathrm{SD}=2.2888$ and $A_{2}$ $=1.9540 \mathrm{D}^{2}$. The values of $a=0.288$ and $m=2.2$ yield $k_{2}=27.675 \times 10^{-3} D^{2}$.

With an SDR $=4.5416$, the bundle area is $A_{\mathrm{b}}=$ $16.1997 D^{2}$. The bundle average permeability is then obtained using the relationship at eq. (16)

$k_{\mathrm{b}}=\frac{1}{A_{\mathrm{b}}}\left(6 A_{1} k_{1}+6 A_{2} k_{2}\right)=0.0219 D^{2}$.

Furthermore,

$\epsilon_{\mathrm{b}}=1-7\left(D / D_{\mathrm{s}}\right)^{2}=0.6606$,

$\mathrm{SD}_{\mathrm{b}}=4\left(7 D+D_{\mathrm{s}}\right) D_{\mathrm{s}}^{2}=2.2382$.

Use of (i) and (ii) yield for the bundle

$k S^{2}=0.1097$.

The bundle average friction factor is then obtained using eq (13) as

$f \operatorname{Re}=\frac{8 \epsilon^{3}}{k S^{2}}=21.02, \quad$ say $C_{1}=21$ (Fig. 2) .

\section{2. $f$ Re correlation}

Darcy's equation is modified to include inertia effects in the form

$-\frac{\mathrm{d} p}{\mathrm{~d} x}=\frac{\mu}{k} \bar{u}+b \rho \bar{u}^{2}$.

The definition of friction factor allows recasting the above equation to

$f_{0}=\frac{C_{1}}{\operatorname{Re}}+C_{2}$.

The porous medium analysis of the foregoing section has yielded the value of $C_{1}=21$ for the present geometry. The turbulent component $C_{2}$ is derived by correlating the experimental results in Fig. 2. Accordingly we get

$f_{0}=21.0 \mathrm{Re}^{-1.0}+5.584 \times 10^{-3}$

with a negligible standard deviation of $2.34 \times 10^{-3}$ representing the pressure losses in both laminar and low Reynolds number turbulent regime: $\operatorname{Re}<30,000$.

It is useful to appreciate that the value of $C_{1}$ in eq. (18) is geometry-dependent. We verified our postulations against the results of Rehme [9] for $\operatorname{Re}=10^{4}$ for hexagonal clusters of $P / D=1.125$ to 1.867 and $W / D$
$=1.142$ to 1.902 for 7 and 19 rod combinations. The laminar $f \operatorname{Re}$ was calculated to vary from 19.385 to 35.668. Application of eq. (18) to the measured friction factor [9] predicted a $C_{2}$ value that varied between $5.233 \times 10^{-3}$ to $5.986 \times 10^{-3}$. In otherwords, eqs. (18) and (19) can be said to display universal characteristics for low Re turbulent axial flow through a bundle.

\subsection{Heat transfer correlation}

The data base for experimental results on heat transfer is not sufficient to establish a very dependable correlation. Yet the results in Fig. 5 indicate a Reynolds analogy type behaviour, with a standard deviation of 1.9 , where we can write

$\mathrm{Nu}_{0}=0.24 \operatorname{Re} f_{0}$

for $\operatorname{Re}<30000$.

To the extent that $f_{0}$ covers wide geometric variation, eq. (20) may be used to rod bundles of different configurations.

\section{Conclusions}

The present experiments conducted on vertical 7-rod bundles in circular shells with forced flow opposing and supporting buoyancy cover a range of $\mathrm{Re}=900$ to 30000 and $\mathrm{Gr}=1.7 \times 10^{3}$ to $6.0 \times 10^{4}$.

We observed that friction factor is increased in downflow and decreased in upflow due to buoyancy. Buoyancy effects at or below $\mathrm{Gr} / \mathrm{Re}= \pm 40$ is insufficient to result in any marked change in thermal transport.

There is no distinct transition from laminar to turbulent regime. The isothermal friction factor is well correlated in the laminar to low Re turbulent range by a porous medium analogy. The laminar component depends upon geometry, and we have illustrated a scheme to derive its value for any bundle, on the basis of porosity that merge $P / D$ and $W / D$ identities. The turbulent component appears universal and has a value of $5.584 \times 10^{-3}$.

\section{Acknowledgement}

This study was performed at IIT, Kharagpur as an Indo-US collaborative project with the Universities of Miami and Michigan-Dearborn with financial support from the National Science Foundation of the United States of America under grant no. INT-8808093. 


\section{Nomenclature}

$A \quad$ cross-sectional area $\left(\mathrm{m}^{2}\right)$,

$A_{\mathrm{b}} \quad$ bundle cross-sectional area $\left(\mathrm{m}^{2}\right)$,

$A_{\mathrm{f}} \quad$ flow or void area $\left(\mathrm{m}^{2}\right)$,

$A_{\mathrm{j}} \quad$ sub-channel cross-sectional area $\left(\mathrm{m}^{2}\right)$,

$D$ rod diameter $(\mathrm{m})$,

$D_{\mathrm{h}} \quad$ hydraulic diameter (m),

$D_{\mathrm{s}} \quad$ shell diameter (m),

$f \quad$ Fanning's friction factor,

Gr Grashof number, $\left.\mathrm{Gr}=\left(g \beta q^{\prime \prime} D^{4}\right) / k_{1} \nu^{2}\right)$,

$h \quad$ heat transfer coefficient $\left(\mathrm{W} / \mathrm{m}^{2} \mathrm{~K}\right)$,

$k$ permeability $\left(\mathrm{m}^{2}\right)$,

$k_{\mathrm{b}} \quad$ bundle permeability $\left(\mathrm{m}^{2}\right)$,

$k_{i} \quad$ sub-channel permeability $\left(\mathrm{m}^{2}\right)$,

$k_{1}$ molecular thermal conductivity of air $(\mathrm{W} / \mathrm{m}$ K),

$L \quad$ length of the test section (m),

$n \quad$ no. of rods in the ring nearest to the shell,

$\dot{m} \quad$ mass flow rate $(\mathrm{kg} / \mathrm{s})$,

$\mathrm{Nu} \quad$ Nusselt number, $\mathrm{Nu}=\frac{h D_{\mathrm{h}}}{k_{1}}$,

$P \quad$ pitch $(\mathrm{m})$,

PDR pitch to rod diameter ratio,

$P_{\mathrm{w}} \quad$ surface area exposed per unit length $(\mathrm{m})$,

$p \quad$ pressure $\left(\mathrm{N} / \mathrm{m}^{2}\right)$,

$\frac{\mathrm{d} p}{\mathrm{~d} x} \quad$ axial pressure gradient $\left(\mathrm{N} / \mathrm{m}^{3}\right)$,

$\left.\frac{\mathrm{d} x}{\mathrm{~d} p}\right|_{\mathrm{b}}$

$\left.\frac{\mathrm{d} p}{\mathrm{~d} x}\right|_{i}$

axial pressure gradient across a bundle $\left(\mathrm{N} / \mathrm{m}^{3}\right)$,

axial pressure gradient across a sub-channel $\left(\mathrm{N} / \mathrm{m}^{3}\right)$,

$\Delta p \quad$ static pressure drop measured over a length $\Delta L\left(\mathrm{~N} / \mathrm{m}^{2}\right)$,

$Q \quad$ electric power in the form of heat (W),

$q^{\prime \prime} \quad$ heat flux on rod surface $\left(\mathrm{W} / \mathrm{m}^{2}\right)$,

$\operatorname{Re} \quad$ Reynolds number, $\operatorname{Re}=\rho u_{\mathrm{av}} D_{\mathrm{h}} / \mu$,

$S \quad$ specific surface area $\left(\mathrm{m}^{-1}\right)$,

SDR shell diameter to rod diameter ratio,

$T_{\mathrm{b}} \quad$ fluid bulk temperature (K),

$T_{\mathrm{w}} \quad$ average rod surface temperature $(\mathrm{K})$,

$u_{\mathrm{av}} \quad$ average velocity through void space $(\mathrm{m} / \mathrm{s})$,

$\bar{u} \quad$ superficial velocity $(\mathrm{m} / \mathrm{s})$,

$w \quad$ wall to rod distance (m), see Fig. 7,

WDR wall to rod diameter ratio.

\section{Greek symbols}

$\beta \quad$ volumetric coefficient of thermal expansion $\left(\mathrm{K}^{-1}\right)$,

$\epsilon$ porosity, $\theta_{0} \quad$ included angle of subchannel, see Fig. 7,

$\rho \quad$ density $\left(\mathrm{kg} / \mathrm{m}^{3}\right)$,

$\mu \quad$ dynamic viscosity (N.s $\left./ \mathrm{m}^{2}\right)$,

$\nu \quad$ kinematic viscosity $\left(\mathrm{m}^{2} / \mathrm{s}\right)$.

Subscripts

0 forced flow.

\section{References}

[1] A.K. Mohanty and K.M. Sahoo, Laminar convection in wall sub-channels and transport rates for finite rod-bundle assemblies by superposition, Nucl. Engrg. Des. 92 (1986) 169-180.

[2] A.K. Mohanty and R. Viskanta, Buoyancy dominated laminar convection and radiation transfer in rod arrays, Int. J. Heat and Fluid Flow 8 (1987) 277-286.

[3] V. Iannello, K.Y. Suh and N.E. Todreas, Mixed convection friction factors and Nusselt numbers in vertical annular and subchannel geometries, Int. J. Heat and Mass Transfer 31 (1988) 2175-2189.

[4] R. Das and A.K. Mohanty, Laminar combined convection in finite circular rod bundles, ASME, J. Heat Transfer 106 (1984) 563-569.

[5] R.W. Benodekar and A.W. Date, Numerical prediction of heat transfer characteristics of fully developed laminar flow through a circular channel containing rod clusters, Int. J. Heat and Mass Transfer 21 (1978) 935-945.

[6] K. Rehme, The structure of turbulent flow through a wall sub-channel of a rod bundle, Nucl. Engrg. Des. 45 (1978) 311-323.

[7] J.G. Bartzis and N.E. Todreas, Turbulence modeling of axial flow in a bare rod bundle, Trans. ASME, J. Heat Transfer 101 (1979) 628-634.

[8] A.K. Mohanty and K.M. Sahoo, Turbulent flow and heat transfer in rod bundle sub-channels, Nucl. Engrg. Des. 106 (1988) 327-344.

[9] K. Rehme, Pressure drop performance of rod bundles in hexagonal arrangements, Int. J. Heat and Mass Transfer 15 (1972) 2499-2517.

[10] S.H. Kim and M.S. El-Genk, Heat transfer experiments for low flow of water in rod bundles, Int. J. Heat and Mass Transfer 32 (1989) 1321-1336.

[11] J.P. Holman, Experimental Methods for Engineers (McGraw-Hill, 1978).

[12] J.W. Yang, Heat transfer and fluid flow in regular rod arrays with opposing flow, in: Fluid Flow and Heat Transfer Over Rod or Tube Bundles, edited by S.C. Yao and P.A. Pfund, ASME Winter Annual Meeting, New York (1979) pp. 149-153.

[13] S. Kakac, R.K. Shah and W. Aung, Convective heat transfer over rod bundles, in: Handbook of Single-Phase Convective Heat Transfer (John Wiley, 1989). 
[14] K. Johannsen, Longitudinal flow over tube bundles in: Low Reynolds Number Flow Heat Exchanger, edited by S. Kakac, R.K. Shah and A.E. Bergles (Hemisphere 1983).

[15] A.E. Scheidegger, The Physics of Flow through Porous Media (University of Toronto Press, 1957).
[16] A.A. Bishop, J.M. Willis and R.A. Markley, Effect of buoyancy on laminar vertical flow friction factors in cylindrical tubes, Nucl. Engrg. Des. 62 (1980) 365-369.

[17] W.M. Kays, Convective Heat and Mass Transfer (McGraw-Hill, 1975). 